\title{
Alumina-mediated dealkylative dimerization of 4-aminobenzyl esters
}

\author{
Yu-Ying Lai, Nai-Ti Lin, Yi-Hung Liu, Yu Wang and Tien-Yau Luh* \\ Department of Chemistry, National Taiwan University, Taipei 106, Taiwan
}

Received 13 January 2007; revised 15 February 2007; accepted 21 February 2007

Available online 27 February 2007

\begin{abstract}
Treatment of 4-aminobenzyl esters with $\mathrm{Al}_{2} \mathrm{O}_{3}$ in DCM at rt afforded the dealkylative dimerized 4,4'-diamino-diphenylmethanes in satisfactory yield. The reaction may proceed via a quinone methide iminium ion intermediate, which may then undergo Michael type addition followed by retro-aldol extrusion of a formaldehyde species.
\end{abstract}

(C) 2007 Elsevier Ltd. All rights reserved.

\section{Introduction}

Diarylmethane derivatives are useful building blocks for the design of supramolecular structures, ${ }^{1}$ polymer synthesis, ${ }^{2}$ as well as biologically active compounds. ${ }^{3}$ The preparation of diarylmethanes can be achieved by numerous procedures such as Friedel-Crafts reaction, ${ }^{4}$ cross coupling reactions, ${ }^{5}$ reduction of the corresponding diaryl ketones ${ }^{6}$ or alcohols, ${ }^{7}$ or disproportionation reactions. ${ }^{8}$ Reaction of $N, N$-dimethyl4-toluidine- $N$-oxide $\mathbf{1}$ in trifluoroacetic acid-triflic acid gives diarylmethane $\mathbf{3}$ as the side product (Scheme 1). ${ }^{9} \mathrm{~A}$ quinone methide iminium ion intermediate $\mathbf{2}$ was suggested.

We recently reported the first helical DNA-like double stranded polymer 5 by ring opening metathesis polymerization ${ }^{10}$ of a bisnorbornene derivative $4 .{ }^{11}$ Because of the presence of the relatively labile aminobenzyl fragment in $\mathbf{5}$, the corresponding single stranded polymer $\mathbf{6}$ is easily obtained by hydrolysis (Scheme 2). ${ }^{11}$ As can be seen from Scheme 2 , the methyl ether $\mathbf{6}$ is obtained exclusively. Presumably, an intermediate similar to $\mathbf{2}$ would be formed during the course of hydrolysis. The chemistry shown in Scheme 2 might be a unique process involving a quinone methide iminium ion intermediate, which would react with methoxide nucleophile in the medium. It is interesting to note that quinone methide derivatives are important intermediates in DNA alkylation and DNA cross-linking reactions. ${ }^{12}$ In order to understand the generality of this novel polymeric system, we have synthesized various kinds of monomers having a range of different linkers to connect the two norbornene units.

During the course of this investigation, we have synthesized bisnorbornene 7 using a diester linkage to tether two aminobenzyl moieties. Attempts to purify $\mathbf{7}$ by column chromatography on neutral alumina or silica gel were unsuccessful. A new dimeric diarylmethane $\mathbf{8}$ was obtained. The structure of 8 was unambiguously proved by X-ray crystallography (Fig. 1 and Table 1).

The isolation of $\mathbf{8}$ was somewhat striking. Presumably, the benzylic $\mathrm{C}-\mathrm{O}$ bond would be extremely labile and cleaved readily under chromatographic separation conditions to give quinone methide iminium ion intermediate 9 . In the absence of other nucleophiles, Michael type addition of another aminobenzyl moiety to $\mathbf{9}$ would yield $\mathbf{1 0}$, which would

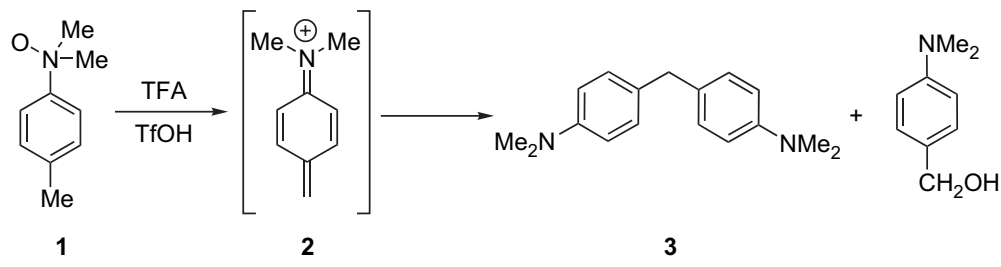

Scheme 1

* Corresponding author. Tel.: +886 223636288; fax: +886 223644971; e-mail: tyluh@ntu.edu.tw 


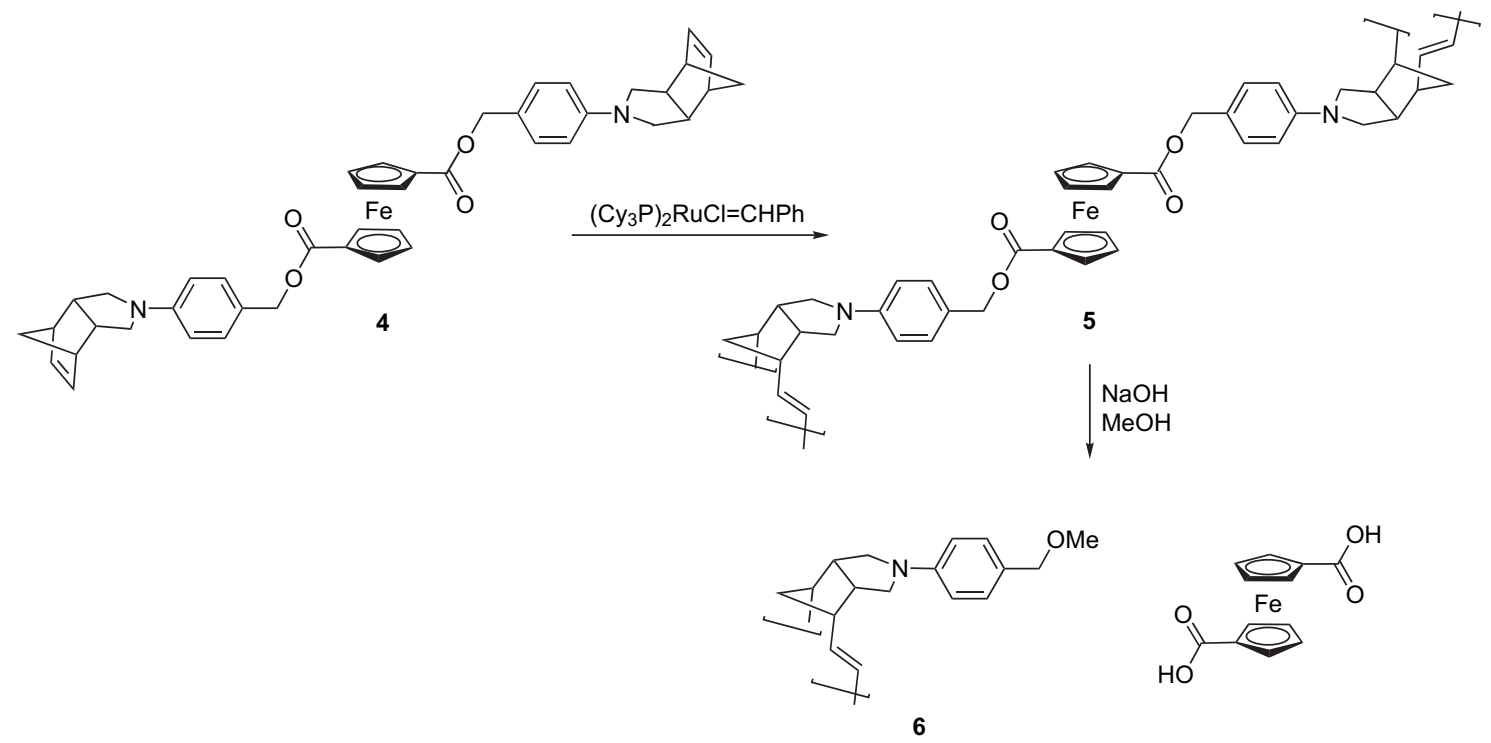

Scheme 2.

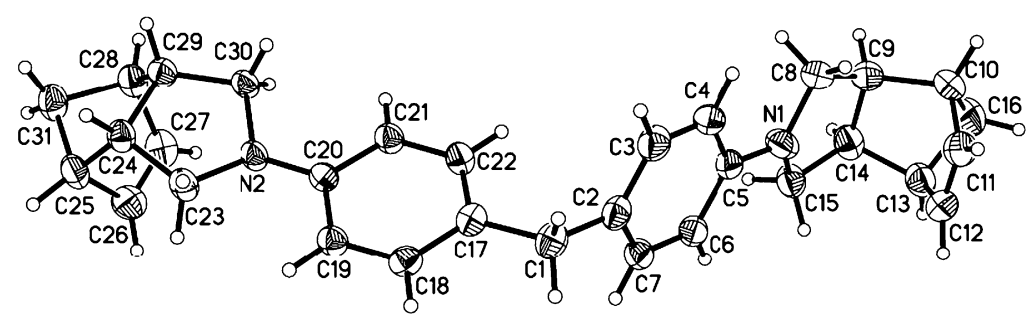

Figure 1. ORTEP structure of 8.
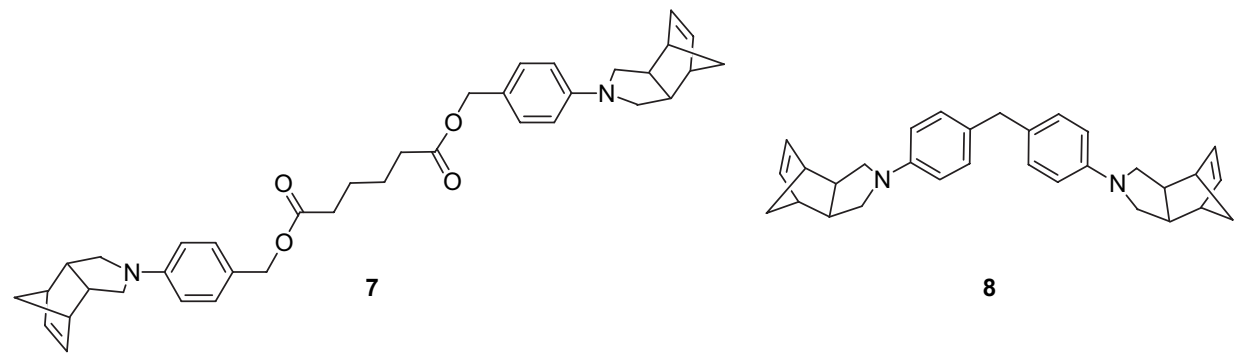

8

then undergo a retro-aldol extrusion of the formaldehyde species to generate $\mathbf{8}$ (Scheme 3 ).

It is envisaged that the reaction might be considered to be a general reaction for 4-aminobenzyl esters. Thus, upon treatment with $\mathrm{Al}_{2} \mathrm{O}_{3}$ in DCM at rt, the crude 12, prepared from the reaction of $\mathbf{1 1}$ and $\mathrm{Ac}_{2} \mathrm{O}$, afforded $\mathbf{8}$ in $40 \%$ yield. When pure $\mathbf{1 2}$ was employed, $\mathbf{8}$ was isolated in $62 \%$ yield. Several different conditions have been tried and the results are outlined in Table 2. It is interesting to note that DCM at $\mathrm{rt}$ was the best condition for this transformation. At refluxing temperature, a significant amount of side products was observed and $\mathbf{8}$ was obtained in $50 \%$ yield. When the reaction was carried out in polar solvents such as THF or DMF, starting compound $\mathbf{1 2}$ was almost completely recovered $(>90)$. It is noteworthy that 8 was obtained in $10 \%$ yield together with a mixture of unidentified products when

EtOAc was used as the reaction medium. When the reaction was carried out in $\mathrm{MeOH}$, the solvent may serve as the nucleophile to quench the quinone methide iminium ion intermediate leading to the corresponding methoxy ether $\mathbf{1 3}$ in 99\% yield (Table 2).

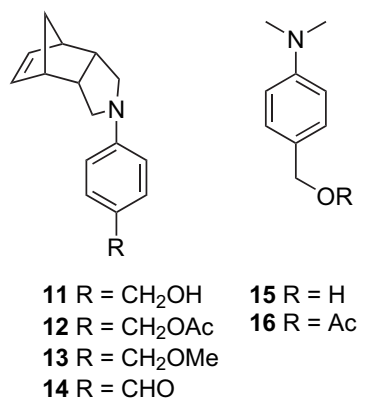

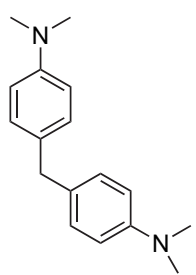

17

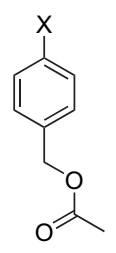

$18 \mathrm{X}=\mathrm{OH}$

$19 \mathrm{X}=\mathrm{OMe}$

$20 \mathrm{X}=\mathrm{Me}$ 
Table 1. Crystal data and structure refinement for $\mathbf{8}$

\begin{tabular}{ll}
\hline Empirical formula & $\mathrm{C}_{31} \mathrm{H}_{34} \mathrm{~N}_{2}$ \\
Formula weight & 434.60 \\
Temperature & $295(2) \mathrm{K}$ \\
Wavelength & $0.71073 \AA$ \\
Crystal system & Monoclinic \\
Space group & $P 2(1) / n$ \\
Unit cell dimensions & $a=19.0940(5) \AA, \alpha=90^{\circ}$ \\
& $b=6.19200(10) \AA, \beta=94.9880(10)^{\circ}$ \\
& $c=19.9310(3) \AA, \gamma=90^{\circ}$ \\
Volume & $2347.52(8) \AA^{3}$ \\
$Z$ & 4 \\
Density (calculated) & $1.230 \mathrm{Mg} / \mathrm{m}^{3}$ \\
Absorption coefficient & $0.071 \mathrm{~mm}$ \\
$F(000)$ & 936 \\
Crystal size & $0.30 \times 0.25 \times 0.15 \mathrm{~mm}^{3}$ \\
Theta range for data collection & $2.05-27.47^{\circ}$ \\
Index ranges & $-24 \leq h \leq 24,-8 \leq k \leq 8,-24 \leq l \leq 25$ \\
Reflections collected & 16,793 \\
Independent reflections & $5337[R($ int $)=0.0369]$ \\
Absorption correction & Semi-empirical from equivalents \\
Max. and min. transmission & 0.990 and 0.974 \\
Refinement method & Full-matrix least-squares on $F^{2}$ \\
Data/restraints/parameters & $5288 / 0 / 299$ \\
Goodness-of-fit on $F^{2}$ & 0.876 \\
Final $R$ indices $[I>2$ sigma $(I)]$ & $R 1=0.0485, w R 2=0.1403$ \\
$R$ indices (all data) & $R 1=0.0926, w R 2=0.1864$ \\
Extinction coefficient & $0.0143(33)$ \\
Largest diff. peak and hole & 0.212 and -0.191 e $\AA^{-3}$ \\
\hline & \\
& \\
&
\end{tabular}
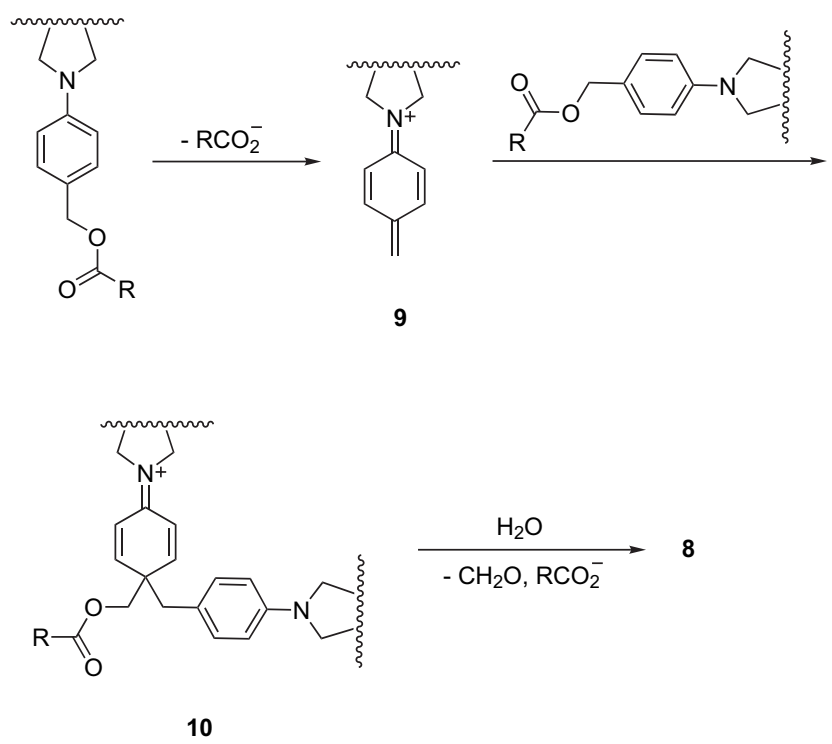

Scheme 3

Table 2. Reaction of 12 with $\mathrm{Al}_{2} \mathrm{O}_{3}$

\begin{tabular}{llc}
\hline Solvent & Temperature & $\%$ Yield \\
\hline THF & $\mathrm{rt}$ & $0^{\mathrm{a}}$ \\
EtOAc & $\mathrm{rt}$ & $10^{\mathrm{b}}$ \\
Toluene & $\mathrm{rt}$ & $2^{\mathrm{c}}$ \\
DMF & $\mathrm{rt}$ & $0^{\mathrm{a}}$ \\
DCM & $\mathrm{rt}$ & 62 \\
DCM & Reflux & 50 \\
MeOH & $\mathrm{rt}$ & $0^{\mathrm{d}}$
\end{tabular}

\footnotetext{
a Starting material was recovered in more than $90 \%$.

b A mixture of unidentified products was obtained in addition to about $25 \%$ of unreacted $\mathbf{1 2}$.

c A mixture of unidentified products was obtained including a significant amount of aldehyde $\mathbf{1 4}$

d Methoxymethyl derivative 13 was obtained in $99 \%$ yield.
}

In a similar manner, the reaction of $\mathbf{1 6}$ under similar conditions in DCM gave dealkylative dimer $\mathbf{1 7}$ in $70 \%$ yield. Attempts to use other substrates having para-hydroxy-, methoxy- or alkyl-substituted benzyl esters 18-20 under similar conditions were unsuccessful. Even in the presence of a base (e.g., DBN), 18 gave a mixture of unidentified products, no desired diarylmethane being detected. Presumably, the reaction may require a strong electron donating substituent to facilitate the leaving of the carboxylate group leading to quinone methide imminium ion intermediate. In addition, the aromatic ring may not be nucleophilic enough to proceed the Michael type addition reaction giving the corresponding diarylmethane derivative.

In summary, we have uncovered an unprecedented dealkylative dimerization of 4-aminobenzyl esters promoted by $\mathrm{Al}_{2} \mathrm{O}_{3}$. The absence of other nucleophiles would be the prerequisite for this reaction. The reaction furnishes a convenient route for the synthesis of dianilinomethane derivatives. Further applications of this protocol are in progress in our laboratory.

\section{Experimental section}

\subsection{Bis-[4-(4-aza-tricyclo[5.2.1.0 $\left.{ }^{2,6}\right]$ dec-8-en-4-yl)]- benzyl hexandioate (7)}

To a DCM solution $(60 \mathrm{~mL})$ of $\mathbf{1 1 3}(2.0 \mathrm{~g}, 8.3 \mathrm{mmol})$ and dimethylaminopyridine (DMAP, $1.0 \mathrm{~g}, 8.3 \mathrm{mmol}$ ) was added slowly adipoyl dichloride $(0.75 \mathrm{~g}, 4.14 \mathrm{mmol})$ in DCM $(20 \mathrm{~mL})$ and the mixture was stirred at $\mathrm{rt}$ for $24 \mathrm{~h}$. Water $(100 \mathrm{~mL})$ was added and the organic layer was separated and washed with brine $(50 \mathrm{~mL})$, dried $\left(\mathrm{MgSO}_{4}\right)$, and filtered. The filtrate was evaporated in vacuo and the oily residue was taken up in DCM/hexane and cooled to $-78{ }^{\circ} \mathrm{C}$. Yellowish solid was precipitated. After filtration, the solid was collected and recrystallized from DCM/hexane to give 7; mp 113$114{ }^{\circ} \mathrm{C}(\mathrm{dec}) ;{ }^{1} \mathrm{H}$ NMR $\left(400 \mathrm{MHz}, \mathrm{CDCl}_{3}\right) \delta 1.50(\mathrm{~d}$, $J=8.2 \mathrm{~Hz}, 2 \mathrm{H}), 1.60$ (d, $J=8.2 \mathrm{~Hz}, 2 \mathrm{H}), 1.62-1.64(\mathrm{~m}, 4 \mathrm{H})$, 2.29 (br s, 4H), 2.88-2.91 (m, 4H), 2.97 (br s, 4H), 3.06$3.07(\mathrm{~m}, 4 \mathrm{H}), 3.18-3.24(\mathrm{~m}, 4 \mathrm{H}), 4.97(\mathrm{~s}, 4 \mathrm{H}), 6.13(\mathrm{~s}, 4 \mathrm{H})$, $6.40(\mathrm{~d}, J=8.5 \mathrm{~Hz}, 4 \mathrm{H}), 7.18(\mathrm{~d}, J=8.5 \mathrm{~Hz}, 4 \mathrm{H}) ;{ }^{13} \mathrm{C} \mathrm{NMR}$ $(100 \mathrm{MHz}) \delta 24.5,34.1,45.6,46.7,50.7,52.3,66.9,111.8$, 122.5, 130.2, 135.9, 147.8, 173.5; IR (KBr) $\nu ~ 2965,2935$, 2848, 1720, 1613, 1526, 1480, 1375, 1260, 1184, 1155, 915, 800, 731; MS (70 eV, EI) $\mathrm{m} / \mathrm{z}(\%) 592(10)\left[\mathrm{M}^{+}\right], 526$ (8), 434 (10), 369 (25), 367 (8), 301 (5), 224 (45), 159 (24), 158 (100), 118 (16); HRMS (EI) $\left(\mathrm{C}_{38} \mathrm{H}_{44} \mathrm{O}_{4} \mathrm{~N}_{2}\right)$ calcd: 592.3301; found: 592.3315 .

\subsection{4-(4-Aza-tricyclo[5.2.1.0 $\left.{ }^{2,6}\right]$ dec-8-en-4-yl)benzyl acetate (12)}

To a solution of $\mathbf{1 1}(2.0 \mathrm{~g}, 8.3 \mathrm{mmol})$ and triethylamine $(3.5 \mathrm{~mL}, 25 \mathrm{mmol})$ in DCM $(15 \mathrm{~mL})$ was added dropwise acetyl chloride $(1.2 \mathrm{~mL}, 9.1 \mathrm{mmol})$ at $0{ }^{\circ} \mathrm{C}$. The mixture was stirred at $\mathrm{rt}$ for $2 \mathrm{~h}$, poured into water $(100 \mathrm{~mL})$, and extracted with DCM $(50 \mathrm{~mL})$. The organic layer was separated, dried $\left(\mathrm{MgSO}_{4}\right)$, filtered, and evaporated in vacuo. The residue was chromatographed on silica gel (hexane/EtOAc 4:1) to afford 12 as a white solid $(2.1 \mathrm{~g}, 92 \%) ; \mathrm{mp} 85-87^{\circ} \mathrm{C} ;{ }^{1} \mathrm{H}$ NMR $\left(400 \mathrm{MHz}, \mathrm{CDCl}_{3}\right) \delta 1.51(\mathrm{~d}, J=8.2 \mathrm{~Hz}, 1 \mathrm{H}), 1.61$ 
(d, $J=8.2 \mathrm{~Hz}, 1 \mathrm{H}), 2.04$ (s, 3H), 2.84-2.87 (m, 2H), 2.88$3.00(\mathrm{~m}, 2 \mathrm{H}), 3.01-3.11(\mathrm{~m}, 2 \mathrm{H}), 3.19-3.24(\mathrm{~m}, 2 \mathrm{H}), 4.98$ $(\mathrm{s}, 2 \mathrm{H}), 6.13(\mathrm{~s}, 2 \mathrm{H}), 6.41(\mathrm{~d}, J=8.6 \mathrm{~Hz}, 2 \mathrm{H}), 7.20(\mathrm{~d}, J=$ $8.6 \mathrm{~Hz}, 2 \mathrm{H}) ;{ }^{13} \mathrm{C} \mathrm{NMR}\left(100 \mathrm{MHz}, \mathrm{CDCl}_{3}\right) \delta 21.3,45.5,46.6$, 50.5, 52.1, 66.9, 111.6, 122.0, 130.1, 135.6, 147.4, 170.9.

\subsection{Bis-\{4-(4-aza-tricyclo[5.2.1.0 $\left.{ }^{2,6}\right]$ dec-8-en-4-yl)- phenyl\}methane (8)}

To a DCM solution $(60 \mathrm{~mL})$ of $\mathbf{1 1}^{13}(2 \mathrm{~g}, 8.3 \mathrm{mmol})$ and DMAP $(1.0 \mathrm{~g}, 8.3 \mathrm{mmol})$ was added slowly acetyl chloride $(0.65 \mathrm{~g}, 8.3 \mathrm{mmol})$ in DCM $(20 \mathrm{~mL})$ and the mixture was stirred at $\mathrm{rt}$ for $24 \mathrm{~h}$. Water $(100 \mathrm{~mL})$ was added and the organic layer was separated and washed with brine, dried $\left(\mathrm{MgSO}_{4}\right)$, and filtered. The solvent was evaporated in vacuo and the oily residue was dissolved in DCM $(40 \mathrm{~mL})$. Neutral alumina ( $45 \mathrm{~g}$ ) was added and the mixture was stirred at $\mathrm{rt}$ for $8 \mathrm{~h}$. After filtration, solvent was removed in vacuo and the residue was recrystallized from DCM/hexane to give $\mathbf{8}$ (0.72 g, 40\%); mp $188-189{ }^{\circ} \mathrm{C}(\mathrm{dec}) ;{ }^{1} \mathrm{H}$ NMR $(400 \mathrm{MHz}$, $\left.\mathrm{CDCl}_{3}\right) \delta 1.50(\mathrm{~d}, J=8.2 \mathrm{~Hz}, 2 \mathrm{H}), 1.59(\mathrm{~d}, J=8.2 \mathrm{~Hz}, 2 \mathrm{H})$, 2.85-2.88 (m, 4H), 2.95 (br s, 4H), 3.03-3.05 (m, 4H), 3.17-3.22 (m, 4H), $3.75(\mathrm{~s}, 2 \mathrm{H}), 6.13(\mathrm{~m}, 4 \mathrm{H}), 6.37$ (d, $J=$ $8.5 \mathrm{~Hz}, 4 \mathrm{H}), 6.99(\mathrm{~d}, J=8.5 \mathrm{~Hz}, 4 \mathrm{H}) ;{ }^{13} \mathrm{C}$ NMR $(100 \mathrm{MHz})$ $40.0,45.5,46.5,50.7,52.2,111.9,129.1,129.3,135.8$, 146.0; IR (KBr) $\nu$ 2964, 2942, 2864, 2833, 1688, 1615, 1518, 1473, 1432, 1369, 1341, 1201, 1186, 1134, 971, 904, 797, 776, 739, 717; MS (70 eV, EI) $\mathrm{m} / \mathrm{z}$ (\%) 434 (100) $\left[\mathrm{M}^{+}\right], 393$ (18), 367 (82), 372 (13), 301 (52); HRMS $\left(\mathrm{FAB}^{+}\right)\left(\mathrm{C}_{31} \mathrm{H}_{34} \mathrm{~N}_{2}\right)$ calcd: 434.2722; found: 434.2725.

From pure 12. Under $\mathrm{N}_{2}$, to a solution of $12(500 \mathrm{mg}$, $1.8 \mathrm{mmol}$ ) in DCM (7 mL) was added $\mathrm{Al}_{2} \mathrm{O}_{3}$ (neutral, $8 \mathrm{~g}$ ) at rt. The mixture was stirred for $20 \mathrm{~h}$ at $\mathrm{rt}$ and then filtered. The $\mathrm{Al}_{2} \mathrm{O}_{3}$ was washed with $\mathrm{DCM} / \mathrm{NEt}_{3}(10: 1)(10 \mathrm{~mL} \times 2)$ and the filtrate was concentrated in vacuo. The residue was chromatographed on silica gel (hexane/EtOAc 7:1) to afford 8 as a white solid (230 mg, 62\%); mp $188-189^{\circ} \mathrm{C}(\mathrm{dec})$.

\subsection{4-(4-Aza-tricyclo[5.2.1.0 $\left.{ }^{2,6}\right]$ dec-8-en-4-yl)benzyl methyl ether (13)}

Under $\mathrm{N}_{2}$, to a solution of $\mathbf{1 2}(50 \mathrm{mg}, 0.2 \mathrm{mmol})$ in $\mathrm{MeOH}$ $\left(5 \mathrm{~mL}\right.$ ) was added $\mathrm{Al}_{2} \mathrm{O}_{3}$ (neutral, $1 \mathrm{~g}$ ) at rt. The mixture was stirred for $20 \mathrm{~h}$ at $\mathrm{rt}$ and then filtered. The $\mathrm{Al}_{2} \mathrm{O}_{3}$ was washed with $\mathrm{DCM} / \mathrm{NEt}_{3}(10: 1)(10 \mathrm{~mL} \times 2)$ and the filtrate was concentrated in vacuo. The residue was passed through a silica gel bed (thickness $=5 \mathrm{~cm}$ ) and washed with (hexane/EtOAc $(7: 1,20 \mathrm{~mL})$. Evaporation of the solvent afforded $\mathbf{1 3}$ as a white solid (44 mg, 99\%); mp 64-65 ${ }^{\circ} \mathrm{C}$ (lit. ${ }^{11 \mathrm{a}} 64-$ $\left.65^{\circ} \mathrm{C}\right) ;{ }^{1} \mathrm{H}$ NMR $\left(400 \mathrm{MHz}, \mathrm{CDCl}_{3}\right) \delta 1.47(\mathrm{dt}, J=1.6$, $8.0 \mathrm{~Hz}, 2 \mathrm{H}), 1.58(\mathrm{dt}, J=1.6,8.0 \mathrm{~Hz}, 2 \mathrm{H}), 2.81-2.84(\mathrm{~m}$, $2 \mathrm{H}), 2.90-2.96(\mathrm{~m}, 2 \mathrm{H}), 2.98-3.06(\mathrm{~m}, 2 \mathrm{H}), 3.15-3.22(\mathrm{~m}$, $2 \mathrm{H}), 3.21(\mathrm{~s}, 3 \mathrm{H}), 4.30(\mathrm{~s}, 2 \mathrm{H}), 6.10(\mathrm{~s}, 2 \mathrm{H}), 6.38(\mathrm{~d}, J=$ $8.6 \mathrm{~Hz}, 2 \mathrm{H}), 7.12(\mathrm{~d}, J=8.6 \mathrm{~Hz}, 2 \mathrm{H}) ;{ }^{13} \mathrm{C}$ NMR $(100 \mathrm{MHz}$, $\left.\mathrm{CDCl}_{3}\right) \delta 45.4,46.4,50.5,52.0,57.2,74.6,111.4,124.3$, 129.1, 135.5, 147.0.

\subsection{Bis-[4-(N,N-dimethylamino)phenyl]methane (17)}

Under $\mathrm{N}_{2}$, to a solution of $\mathbf{1 6}(500 \mathrm{mg}, 1.8 \mathrm{mmol})$ in DCM $\left(12 \mathrm{~mL}\right.$ ) was added $\mathrm{Al}_{2} \mathrm{O}_{3}$ (neutral, $14 \mathrm{~g}$ ) at rt. The mixture was stirred for $20 \mathrm{~h}$ at $\mathrm{rt}$ and then filtered. The $\mathrm{Al}_{2} \mathrm{O}_{3}$ was washed with $\mathrm{DCM} / \mathrm{NEt}_{3}(10: 1,10 \mathrm{~mL} \times 2)$ and the filtrate was concentrated in vacuo to give the residue, which was chromatographed on silica gel (hexane/EtOAc 7:1) to afford 17 as a white solid $(220 \mathrm{mg}, 70 \%)$; mp $82-83{ }^{\circ} \mathrm{C}$ (lit. ${ }^{14} 86.5-$ $\left.87{ }^{\circ} \mathrm{C}\right) ;{ }^{1} \mathrm{H}$ NMR $\left(400 \mathrm{MHz}, \mathrm{CDCl}_{3}\right) \delta 2.87(\mathrm{~s}, 12 \mathrm{H}), 3.79$ (s, $2 \mathrm{H}), 6.66(\mathrm{~d}, J=8.8 \mathrm{~Hz}, 2 \mathrm{H}), 7.03(\mathrm{~d}, J=8.8 \mathrm{~Hz}, 2 \mathrm{H}) ;{ }^{13} \mathrm{C}$ NMR $\left(100 \mathrm{MHz}, \mathrm{CDCl}_{3}\right) \delta 39.9,40.9,112.8,129.2,130.1$, 148.8. HRMS $\left(\mathrm{FAB}^{+}\right)\left(\mathrm{C}_{17} \mathrm{H}_{22} \mathrm{~N}_{2}\right)$ calcd: 254.1783 ; found: 254.1780 .

\section{Acknowledgements}

This work is supported by the National Science Council and the National Taiwan University.

\section{Supplementary data}

Supplementary data associated with this article can be found in the online version, at doi:10.1016/j.tet.2007.02.094.

\section{References and notes}

1. (a) Ma, J. C.; Dougherty, D. A. Chem. Rev. 1997, 97, 1303; (b) Jäfer, R.; Vögtle, F. Angew. Chem., Int. Ed. 1997, 36, 930.

2. (a) Bruno, J. G.; Chang, M. N.; Choi-Sledeski, Y. M.; Green, D. M.; MaGarry, D. G.; Regan, J. R.; Volz, F. A. J. Org. Chem. 1997, 62, 5174; (b) Wllkinson, J. A.; Rossington, S. B.; Leonard, J.; Hussain, N. Tetrahedron Lett. 2004, 45, 5481; (c) Maciejewska, M.; Gawdzik, B. J. Appl. Polym. Sci. 2005, $95,863$.

3. (a) Stanchev, S.; Rakovska, R.; Berova, N.; Snatzke, G. Tetrahedron: Asymmetry 1995, 6, 183; (b) Ku, Y.-Y.; Patel, R. P.; Sawick, D. P. Tetrahedron Lett. 1996, 37, 1949; (c) de Lang, R.-J.; van Hooijdonk, M. J. C. M.; Brandsma, L.; Kramer, H.; Seinen, W. Tetrahedron 1998, 54, 2953; (d) Prat, L.; Mojovic, L.; Levacher, V.; Dupas, G.; Quéguiner, G.; Bourguignon, J. Tetrahedron: Asymmetry 1998, 9, 2509; (e) Rische, T.; Eilbracht, P. Tetrahedron 1999, 55, 1915.

4. For examples: (a) Coq, B.; Gourves, V.; Figueras, F. Appl. Catal., A 1993, 100, 69; (b) Yadav, G. D.; Thorat, T. S.; Khumbar, P. S. Tetrahedron Lett. 1993, 34, 529; (c) Cseri, T.; Bekassy, S.; Figueras, F.; Rizner, S. J. Mol. Catal. A 1995, 98, 101; (d) Tanabe, K.; Holderich, W. F. Appl. Catal., A 1999, 181, 339; (e) de la Cruz, M. H. C.; da Silva, J. F. C.; Lachter, E. R. Appl. Catal., A: Gen. 2003, 245, 377; (f) Sun, H.-B.; Hua, R.; Yin, Y. Tetrahedron Lett. 2006, 47, 2291.

5. For examples, see: (a) Hossain, K. M.; Takagi, K. Chem. Lett. 1999, 1241; (b) García Martínez, A.; Osío Barcina, J.; Colorado Heras, M.; de Fresno Cerazo, A. Org. Lett. 2000, 2, 1377; (c) Itami, K.; Mineno, M.; Kamei, T.; Yoshida, J.-I. Org. Lett. 2002, 4, 3635; Park, C.-M.; Sun, C.; Olejniczak, E. T.; Wilson, A. E.; Meadows, R. P.; Betz, S. F.; Elmore, S. W.; Fesik, S. W. Bioorg. Med. Chem. Lett. 2005, 15, 771; (d) Frisch, A. C.; Shaikh, N.; Zapf, A.; Beller, M. Angew. Chem., Int. Ed. 2002, 41, 4056; (e) Pirrung, M. C.; Wedel, M.; Zhao, Y. Synlett 2002, 143; (f) Langle, S.; Abarbri, M.; Duchêne, A. Tetrahedron Lett. 2003, 44, 9255; (g) Nobre, S. M.; Monteiro, A. L. Tetrahedron Lett. 2004, 45, 8225; (h) Park, S. Y.; Kang, M.; Yie, J. E.; Kim, J. M.; Lee, I.-M. Tetrahedron Lett. 2005, 46, 2849. 
6. (a) Popielarz, R.; Arnold, D. R. J. Am. Chem. Soc. 1990, 112, 3068; (b) Lee, W. Y.; Park, W. Y.; Kim, Y. D. J. Org. Chem. 1992, 57, 4074; (c) Lee, W. Y.; Park, C. H.; Kim, H. J.; Kim, S. J. J. Org. Chem. 1994, 59, 878; (d) Gadhwal, S.; Baruah, M.; Sandhu, J. S. Synlett 1999, 1573; (e) Hicks, L. D.; Han, J. K.; Fry, A. J. Tetrahedron Lett. 2000, 41, 7817; (f) Studer, M.; Burkhardt, S.; Indolese, A. F.; Blaser, H.-U. Chem. Commun. 2000, 1327; (g) Chandrasekhar, S.; Reddy, C. R.; Babu, B. N. J. Org. Chem. 2002, 67, 9080; (h) Toyota, S.; Nakagawa, T.; Kotani, M.; Oki, M.; Uekusa, H.; Ohashi, Y. Tetrahedron 2002, 58, 10345; (i) Mahmoodi, N. O.; Salehpour, M. J. Heterocycl. Chem. 2003, 40, 875; (j) Hatano, B.; Tagaya, H. Tetrahedron Lett. 2003, 44, 6331; (k) Tremont, S. J.; Lee, L. F.; Huang, H.-C.; Keller, B. T.; Banerjee, S. C.; Both, S. R.; Carpenter, A. J.; Wang, C.-C.; Garland, D. J.; Huang, W.; Jones, C.; Koeller, K. J.; Kolodziej, S. A.; Li, J.; Manning, R. E.; Mahoney, M. W.; Miller, R. E.; Mischke, D. A.; Rath, N. P.; Fletcher, T.; Reinhard, E. J.; Tollefson, M. B.; Vernier, W. F.; Wagner, G. M.; Rapp, S. R.; Beaudry, J.; Glenn, K.; Regina, K.; Schuh, J. R.; Smith, M. E.; Trivedi, J. S.; Reitz, D. B. J. Med. Chem. 2005, 48, 5837; (1) Zaccheria, F.; Ravasio, N.; Ercoli, M.; Allegrini, P. Tetrahedron Lett. 2005, 46, 7743.

7. (a) Miyai, T.; Onishi, Y.; Baba, A. Tetrahedron Lett. 1998, 39, 6291; (b) Bringmann, G.; Pabst, T.; Henschel, P.; Michel, M. Tetrahedron 2001, 57, 1269; (c) Waterlot, C.; Hasiak, B.; Couturier, D.; Rigo, B. Tetrahedron 2001, 57, 4889; (d) Brousmiche, D. W.; Xu, M.; Lukeman, M.; Wan, P. J. Am. Chem. Soc. 2003, 125, 12961; (e) Barda, D. A.; Wang, Z.-Q.; Britton, T. C.; Henry, S. S.; Jagdmann, G. E.; Coleman, D. S.; Johnson, M. P.; Andis, S. L.; Schoepp, D. D. Bioorg. Med. Chem. Lett. 2004, 14, 3099; (f) Okimoto, M.; Takahashi, Y.; Nagata, Y.; Satoh, M.; Sueda, S.; Yamashina, T. Bull. Chem. Soc. Jpn. 2004, 77, 1405; (g) Long, Y.-Q.; Jiang, X.-H.; Dayam, R.; Sanchez, T.; Shoemaker, R.; Sei, S.;
Neamati, N. J. Med. Chem. 2004, 47, 2561; (h) Wu, X.; Mahalingam, A. K.; Alterman, M. Tetrahedron Lett. 2005, 46, 1501; (i) Ihmels, H.; Meiswinkel, A.; Mohrschladt, C. J.; Otto, D.; Waidelich, M.; Towler, M.; White, R.; Albrecht, M.; Schnurpfeil, A. J. Org. Chem. 2005, 70, 3929.

8. (a) Bartlett, P. D.; McCollum, J. D. J. Am. Chem. Soc. 1956, 78, 1441; (b) Climent, M. J.; Corma, A.; Garcia, S.; Iborra, S.; Primo, J. Recl. Trav. Chim. Pays-Bas 1991, 110, 275; (c) Zhu, Z.; Espenson, J. H. J. Org. Chem. 1996, 61, 324; (d) Gautret, P.; El-Ghammarti, S.; Legrand, A.; Couturier, D.; Rigo, B. Synth. Commun. 1996, 26, 707; (e) Waterlot, C.; Couturier, D.; Backer, M. D.; Rigo, B. Can. J. Chem. 2000, 78, 1242; (f) Hatano, B.; Kadokawa, J.-I.; Tagaya, H. Tetrahedron Lett. 2002, 43, 5859; (g) Harig, M.; Neumann, B.; Stammler, H.-G.; Kuck, D. Eur. J. Org. Chem. 2004, 69, 2381; (h) L'Hermite, N.; Giraud, A.; Provot, O.; Peyrat, J.-F.; Alami, M.; Brion, J.-D. Tetrahedron 2006, 62, 11994.

9. Lindsay Smith, J. R.; Linford, J. M.; McKeer, L. C.; Morris, P. M. J. Chem. Soc., Perkin Trans. 2 1984, 1099.

10. Schwab, P.; Grubbs, R. H.; Ziller, J. W. J. Am. Chem. Soc. 1996, $118,100$.

11. (a) Yang, H.-C.; Lin, S.-Y.; Yang, H.-c.; Lin, C.-L.; Tsai, L.; Huang, S.-L.; Chen, I.-W. P.; Chen, C.-h.; Jin, B.-Y.; Luh, T.Y. Angew. Chem., Int. Ed. 2006, 45, 726; (b) Yang, H.-C.; Lin, S.-M.; Liu, Y.-H.; Wang, Y.; Chen, M.-M.; Sheu, H.-S.; Tsou, D.-L.; Lin, C.-H.; Luh, T.-Y. J. Organomet. Chem. 2006, 691, 3196.

12. For a recent review, see: Wang, P.; Song, Y.; Zhang, L.; He, H.; Zhou, X. Curr. Med. Chem. 2005, 12, 2893.

13. Lin, W.-Y.; Murugesh, M. G.; Sudhakar, S.; Yang, H.-C.; Tai, H.-C.; Chang, C.-S.; Liu, Y.-H.; Wang, Y.; Chen, I.-W. P.; Chen, C.-h.; Luh, T.-Y. Chem.-Eur. J. 2006, 12, 324.

14. Sakakibara, T.; Dogomori, Y.; Tsuzuki, Y. Bull. Chem. Soc. Jpn. 1979, 52, 3592. 